\title{
G. Dorta
}

\section{Helicobacter pylori Detection Revisited}

In the present issue of Endoscopy, Fruehauf et al. [1] report the results of a study comparing the gastroscopic real-time ${ }^{13} \mathrm{C}$-urea breath test with the rapid urease test.

Since the recognition of the pathophysiological role of Helicobacter pylori in peptic ulcer diseases, gastric carcinoma and mucosaassociated lymphoid tissue lymphoma, hundreds of articles have been published on $H$. pylori tests. $H$. pylori infection can be detected by invasive and noninvasive methods. It can be recognized in gastric mucosa specimens, in feces, in urine, and in blood. Several test methods are available for research and clinical purposes, including: histological staining, microbiological culture, polymerase chain reaction, enzyme-linked immunoabsorbent assay, immunoblotting, and urease-based tests [2,3]. For clinical purposes, the noninvasive ${ }^{13} \mathrm{C}$ breath test and biopsy-based rapid urease test are the ones most frequently used. Today, these two tests are highly sensitive (86-98\%) and specific (90-100\%); they are easy to carry out and are relatively inexpensive.

Before taking a critical look at the above-mentioned paper, we should ask whether there is actually any need for new clinical $H$. pylori tests, and what requirements new tests would have to meet in order to make them superior to the older tests. To achieve improved sensitivity and specificity is not easy to accomplish, as the sensitivity and the specificity of the available tests are already quite high. One primary goal would be the development of a reliable $H$. pylori test that would detect infection in patients receiving antisecretory therapy, or in the presence of blood in the stomach. Another goal might be to develop tests that are cheaper, more convenient, and faster than the available ones. All of these aspects need to be taken into account in discussing the paper on gastroscopic breath testing by Fruehauf et al. [1].
In a prospective, randomized, and controlled study, Fruehauf and colleagues investigated the feasibility of $H$. pylori breath tests during gastroscopy and compared this procedure with the rapid urease test. The validated sensitivity and specificity of the gastroscopic breath test (GBT) were $97.8 \%$ and $96.1 \%$, respectively. The gastroscopic ${ }^{13} \mathrm{C}$ breath test was carried out during upper gastrointestinal endoscopy. After inspection of the gastric mucosa, the ${ }^{13} \mathrm{C}$-urea solution was injected into the stomach through the scope. Breath samples, aspirated via a nasal cannula, were continuously analyzed using molecular correlation spectroscopy. The result of the GBT was available after a mean of $14 \mathrm{~min}$, compared with $19 \mathrm{~h}$ for the rapid urease test. Gastroscopy with GBT took slightly longer to perform and prolonged the occupancy of the endoscopy room by 5-6 min in comparison with rapid urease testing. The authors emphasize that GBT is a noninvasive test and can be used without risks in patients with bleeding disorders, those receiving anticoagulation therapy, those taking nonsteroidal anti-inflammatory drugs (NSAIDs), and in patients lacking endocarditis prophylaxis. There are no contraindications to GBT and only a few restrictions regarding its application (inadequate nasal breathing, severe malabsorption, low-output circulation states, severely reduced liver function, and severe respiratory diseases). The authors conclude that the use of GBT is particularly attractive in patients in whom biopsy sampling is contraindicated.

In their study, $20 \%$ of the patients assigned to the arm receiving the rapid urease test had to be excluded because of bleeding disorders, anticoagulation, NSAID intake, and lack of endocarditis prophylaxis. The authors regard the GBT as being potentially useful in these patients. However, following the guidelines promulgated by the American Society of Gastrointestinal Endoscopy 
[4, 5], neither NSAID intake nor a lack of endocarditis prophylaxis are contraindications to gastric biopsies. In the study under discussion, therefore, the number of indications for GBT would be considerably reduced. As an alternative, $H$. pylori testing in patients with bleeding disorders or receiving anticoagulation therapy can always be carried out using the classic ${ }^{13} \mathrm{C}$ breath test. Furthermore, rapid urease testing could also be carried out with material obtained with a cytology brush, even in patients with severe bleeding disorders.

Another potential advantage of GBT postulated by the authors is a reduced risk of transmitting infectious diseases. It is true that GBT does not require biopsies, but GBT is still an invasive test and the risk of transmission is not reduced to zero, since the endoscope may be contaminated by contagious infectious agents.

What about the other requirements that need to be met by new $H$. pylori tests? The sensitivity and specificity of GBT cannot be better than those of the classical ${ }^{13} \mathrm{C}$ breath test, since the principle of GBT is the same. In addition, patients undergoing upper gastrointestinal endoscopy under conscious sedation often receive oxygen therapy, and the way in which oxygen therapy might affect the results of GBT is not known.

In my opinion, the GBT does not resolve the problem of detecting $H$. pylori in patients who are receiving antisecretory treatment or in patients with acute upper gastrointestinal bleeding.

Is GBT inexpensive? Unfortunately, no data are provided regarding the costs of GBT in comparison with those of rapid urease testing. I am not certain whether we can agree with the authors' statement that the two tests generate comparable costs. The GBT is probably expensive - with the ${ }^{13} \mathrm{C}$-urea solution, the analysis equipment, the need for an additional staff member to monitor and handle the analysis equipment, and a longer period of occupancy of the endoscopy room by the patient. Whether we like it or not, financial aspects and economic pressures have become increasingly important in medicine, and each new medical procedure that is proposed also has to undergo economic assessment.
Is GBT easy to carry out? The problem begins with the principle of the test - an ordinary swallow has to be replaced by a gastroscopic procedure. Could the patient not swallow ${ }^{13} \mathrm{C}$-urea after the endoscopy as well? GBT requires the BreathID device to be installed in the endoscopy room, and sampling devices. Continuous breath sampling for 15 min must be ensured. The analytical procedure has to be monitored for correct functioning during the test. To me, the GBT procedure thus appears to be rather inconvenient. In contrast, rapid urease testing is a very simple and reliable procedure.

Finally, one real advantage of GBT is evident even to critics: the results are rapidly available and can be attached to the endoscopy report. This allows time and costs to be saved by avoiding the need to send additional reports of the $H$. pylori results to the patient's attending physician.

Nevertheless, I am not certain whether the gastroscopic breath test appears to solve any of the existing problems in the search for $H$. pylori. The place of the test in the gastroenterological armamentarium therefore appears unclear and will need to be more clearly defined. I remain skeptical.

\section{References}

${ }^{1}$ Fruehauf H, Lindenmann NC, Volkart K, Bauerfeind P, Fried M. Gastroscopic real-time ${ }^{13} \mathrm{C}$-urea breath test. Endoscopy 2005; 37: $527-531$

${ }^{2}$ Makristathis A, Hirschl AM, Lehours P, Megraud F. Diagnosis of Helicobacter pylori infection. Helicobacter 2004; 9 (Suppl 1): 7-14

${ }^{3}$ Mitchell H, Megraud F. Epidemiology and diagnosis of Helicobacter pylori infection. Helicobacter 2002; 7 (Suppl 1): 8-16

${ }^{4}$ Eisen GM, Baron TH, Dominitz JA et al. Guideline on the management of anticoagulation and antiplatelet therapy for endoscopic procedures. American Society for Gastrointestinal Endoscopy. Gastrointest Endosc 2002; 55: 775-779

${ }^{5}$ Hirota WK, Petersen K, Baron TH et al. Guidelines for antibiotic prophylaxis for GI endoscopy. Standards of Practice Committee of the American Society for Gastrointestinal Endoscopy. Gastrointest Endosc 2003; 58 : $475-482$ 